\title{
ACTIVE LOCALIZATION OF VIRTUAL SOUNDS
}

\author{
J. M. Loomis, C. Hebert, and J. G. Cicinelli \\ University of California \\ Santa Barbara, California
}

We describe a virtual sound display built around a $12 \mathrm{MHz} 80286$ microcomputer and specialpurpose analog hardware. The display implements most of the primary cues for sound localization in the ear-level plane. Static information about direction is conveyed by interaural time differences and, for frequencies above $1800 \mathrm{~Hz}$, by head sound shadow (interaural intensity differences) and pinna sound shadow. Static information about distance is conveyed by variation in sound pressure (first power law) for all frequencies, by additional attenuation in the higher frequencies (simulating atmospheric absorption), and by the proportion of direct to reverberant sound. When the user actively locomotes, the changing angular position of the source occasioned by head rotations provides further information about direction and the changing angular velocity produced by head translations (motion parallax) provides further information about distance. Judging both from informal observations by users and from objective data obtained in an experiment on homing to virtual and real sounds, we conclude that simple displays such as this are effective in creating the perception of external sounds to which subjects can home with accuracy and ease. 